\title{
Performance of Primary Angioplasty for STEMI during the COVID-19 Outbreak
}

\author{
Surya Dharma, MD, PhD, FIHA, FAPSIC, FESC, FSCAI ${ }^{1}$ Iwan Dakota, MD, PhD, FIHA, FESC, FACC ${ }^{2}$ \\ Isman Firdaus, MD, PhD, FIHA, FACC, FESC, FSCAI ${ }^{2}$ Siska Suridanda Danny, MD, FIHA ${ }^{2}$ \\ Dian Zamroni, MD, FIHA ${ }^{2}$ Ardi Yudha, MD $^{3}$ Agus Susanto, CVRN ${ }^{4}$ \\ Bambang Budi Siswanto, MD, PhD, FIHA ${ }^{2}$
}

1 Department of Cardiology and Vascular Medicine, Faculty of
Medicine, University of Indonesia, Indonesian Cardiovascular
Research Center, National Cardiovascular Center Harapan Kita,
Jakarta, Indonesia
2 Department of Cardiology and Vascular Medicine, Faculty of
Medicine, University of Indonesia, National Cardiovascular Center
Harapan Kita, Jakarta, Indonesia
${ }^{3}$ Indonesian Cardiovascular Research Center, National Cardiovascular
Center Harapan Kita, Jakarta, Indonesia
${ }^{4}$ Catheterization Laboratory Nurse Manager, National Cardiovascular
Center Harapan Kita, Jakarta, Indonesia

Address for correspondence Surya Dharma, MD, PhD, FIHA, FAPSIC, FESC, FSCAI, Department of Cardiology and Vascular Medicine, Faculty of Medicine, University of Indonesia, Indonesian Cardiovascular Research Center, National Cardiovascular Center Harapan Kita, Jl S Parman Kav 87, Slipi, Jakarta Barat, 11420, Jakarta, Indonesia (e-mail: drsuryadharma@yahoo.com).

Int J Angiol 2021;30:148-154.

\section{Abstract \\ Keywords \\ - primary angioplasty \\ - performance measures \\ - COVID-19 outbreak}

There has been concern whether the declining cases of ST-segment elevation myocardial infarction (STEMI) during the coronavirus disease 2019 (COVID-19) outbreak associate with primary angioplasty performance.

We assessed the performance of primary angioplasty in a tertiary care hospital in Jakarta, Indonesia, by comparing the door-to-device (DTD) time and thrombolysis in myocardial infarction (TIMI) flow after angioplasty between two periods of admission: during the outbreak of COVID-19 (March 1 to May 31, 2020) and before the outbreak (March 1, to May 31, 2019).

Overall, there was a relative reduction of $44 \%$ for STEMI admission during the outbreak $(n=116)$ compared with before the outbreak $(N=208)$. Compared with before the outbreak period $(n=141)$, STEMI patients who admitted during the outbreak and received primary angioplasty $(n=70)$ had similar median symptom onset-to-angioplasty center admission (360 minutes for each group), similar to radial access uptake (90 vs. $89.4 \%, p=0.88$ ) and left anterior descending infarct-related artery ( 54.3 vs. $58.9 \%, p=0.52$ ). The median DTD time and total ischemia time were longer (104 vs. 81 minutes, $p<0.001$, and 475.5 vs. 449 minutes, $p=0.43$, respectively). However, the final achievement of TIMI 3 flow was similar ( 87.1 vs. $87.2 \%)$, and so was the inhospital mortality (5.7 vs. $7.8 \%$ ).

During the COVID-19 outbreak, we found a longer DTD time for primary angioplasty, but the achievement of final TIMI 3 flow and in-hospital mortality were similar as compared with before the outbreak. Thus, primary angioplasty should remain the standard of care for STEMI during the COVID-19 outbreak. published online April 20, 2021 (c) 2021. International College of Angiology. All rights reserved. Thieme Medical Publishers, Inc., 333 Seventh Avenue, 18th Floor, New York, NY 10001, USA
DOI https://doi.org/ $10.1055 / \mathrm{s}-0041-1727133$. ISSN 1061-1711. 
During the coronavirus disease 2019 (COVID-19) outbreak, the hospital admissions for acute coronary syndrome (ACS) including ST-segment elevation myocardial infarction (STEMI) have significantly declined. ${ }^{1,2}$ Whether the declining cases of STEMI during the COVID-19 outbreak associate with the performance of primary percutaneous coronary intervention (PCI) for STEMI has not much been studied. The aim of the present analysis is to investigate the performance of primary PCI for STEMI during the early months of the COVID-19 outbreak in Jakarta, Indonesia, which at the time of analysis, Jakarta, is the epicenter of the COVID-19 outbreak in the country.

\section{Methods}

\section{Study Design, Population, and Settings}

We performed a retrospectively analysis of the Jakarta Acute Coronary Syndrome (JAC) registry, which is an observational, prospective registry, collecting data on consecutive patients with ACS (STEMI, non-STEMI, and unstable angina pectoris) who are admitted to the emergency department (ED) of a receiving primary $\mathrm{PCI}$ hospital. The hospital is a tertiary care academic cardiovascular center, which is located at Jakarta, Indonesia, and is hosting the STEMI network in the metropolitan area. Details of the JAC registry have been previously described. ${ }^{3,4}$ Data collection and accuracy were evaluated on a regular basis, and all authors wrote the manuscript and aware for the completeness and data accuracy, and analyses. The study approved by the institutional review board of the National Cardiovascular Center Harapan Kita, Jakarta. In this registry, the informed consent was waived, and data were analyzed anonymously.

In this study, we included all consecutive patients with STEMI who were admitted to the hospital during the COVID19 outbreak (March 1, 2020 to May 31, 2020) and before the outbreak (March 1, 2019 to May 31, 2019).

We compared the clinical and angiographic characteristics of patients with STEMI between the two periods. The comparison period (March 1, 2019 to May 31, 2019) was selected to appropriately describe the profile of the realworld STEMI patients admitted to the hospital within the similar observation period used during the COVID-19 outbreak.

\section{STEMI Network in Jakarta}

The JAC registry is routinely being used as the performance measures for the regional STEMI network in the metropoli$\tan$ area of Jakarta, Indonesia. ${ }^{3-5}$ The STEMI network in Jakarta is hosted by the ED team of our hospital, offering 24/7 primary PCI service, and is the largest cardiovascular center in the region. In 2019, the hospital performs $\sim 600$ primary PCI procedures. Currently, there are $\sim 100$ health centers involved in the regional STEMI network in Jakarta and the surrounding area.

\section{Primary PCI Procedure}

Primary PCI procedure in our hospital has been previously described. ${ }^{6}$ In brief, all patients were pretreated with 160 to
$320 \mathrm{mg}$ acetylsalicylic acid and $600 \mathrm{mg}$ clopidogrel or $180 \mathrm{mg}$ ticagrelor (peroral). Unfractionated heparin (100 IU/kg) was administered intravenously in the catheterization laboratory after sheath insertion. During primary PCI, an initial coronary angiogram was performed to assess the infarct-related artery (IRA), and baseline thrombolysis in myocardial infarction (TIMI) flow. After primary PCI, the final TIMI flow was evaluated using standard projection.

\section{COVID-19 Outbreak in Jakarta}

Jakarta covers $662 \mathrm{~km}^{2}$ (256 sq mi) of land area, and $\sim 11$ million population reside with the population density of $\sim$ 15,000 people $/ \mathrm{km}^{2}{ }^{7}$ The first two cases of COVID-19 in Indonesia were confirmed in Jakarta in March 2, 2020, which were increased to 2902 in April 18, 2020, and reached 3,832 cases by April 26, 2020, and making Jakarta as the epicenter of COVID-19 outbreak in the country. ${ }^{8}$

\section{Data Collection and Quality Control}

Data were collected from the JAC registry electronic dataset including sex and age of the patient, diagnosis, coronary artery disease risk factors, Killip classifications, TIMI risk score, source of referral, blood test results, left ventricular ejection fraction from echocardiography, final TIMI flow after primary $\mathrm{PCI}$, ischemic time metrics for primary PCI, and in-hospital mortality. All data were checked for accuracy, and if the main data was incomplete, the data were checked again and then completed.

\section{Outcome Measures}

The primary outcome measure was the performance of primary PCI as defined by the door-to-device (DTD) time, and achievement of final TIMI 3 flow after PCI. Secondary outcomes included the symptom onset-to-PCI hospital admission time, total ischemic time, and in-hospital mortality.

\section{Study Definitions}

The diagnosis of STEMI was made based on the presence of ischemic symptoms (>20 minutes) and persistent ST-segment elevation in at least two contiguous leads, a new left bundle-branch block, or a true posterior myocardial infarction confirmed by posterior leads from the electrocardiography (ECG). ${ }^{9}$

DTD time was calculated as time period between ED admission at the PCI center and first device introduction in an attempt to reopen the occluded IRA. ${ }^{9}$ Total ischemic time was defined as time period between start of the symptom (symptom onset) and first device introduction during primary PCI after the coronary guide wire has crossed the culprit lesion. ${ }^{10}$ Symptom-to-PCI center admission time was defined as time from the first symptom onset to admission at the ED of the PCI center.

\section{Statistical Analysis}

Patient characteristics and the study outcomes between the two admission times (during and before the COVID-19 outbreak) were compared with the use of Mann-Whitney 
$U$ test for continuous variables and chi-squared or Fisher's exact tests for categorical variables.

All statistical analyses were performed with the use of the IBM SPSS statistical package, version 25.0 (Chicago, IL). All tests were two-tailed, and $p$-values of less than 0.05 were considered statistically significance.

\section{Results}

\section{Study Population}

Of 324 patients with acute STEMI recorded, 116 patients were admitted during the COVID-19 outbreak, and 208 patients were admitted before the COVID-19 outbreak period. Of these, 70 and 141 patients who received primary PCI were included the final analysis (-Fig. 1).

\section{Patient Characteristics}

Characteristics of patients who received primary PCI between the two periods are shown in -Table 1. The median age was 55 years in the group of during the COVID-19 outbreak and 54 years in the group of before the outbreak; the percentage of male patients was $\sim 90 \%$ in both groups. During the COVID-19 outbreak period, the admission of STEMI patients through interhospital transfer dropped by $74 \%$ (24 vs. 94 patients), the percentage of smoker and median creatinine level at admission were lower (35.7 vs.
$65.2 \%, p<0.001$; and 0.94 vs. $1.03 \mathrm{mg} / \mathrm{dl}, p=0.006$, respectively), but the uptake of radial access and left anterior descending IRA were similar as compared with before the COVID-19 outbreak period (90 vs. $89.4 \%$, and 54.3 vs. $58.9 \%$, respectively).

\section{Outcomes}

Study outcomes are displayed in - Table 2. Compared with the period before the COVID-19 outbreak, patients with STEMI who were admitted to the PCI hospital during the COVID-19 outbreak had longer median DTD time (104 vs. 81 minutes, $p<0.001$ ), and longer total ischemia time (475.5 vs. 449 minutes, $\mathrm{P}=\mathrm{NS}$ ). However, the symptom onset-to-PCI center admission time was similar (360 minute for each group), and so were the achievement of TIMI 3 flow after primary PCI (87.1 vs. 87.2\%), and inhospital mortality (5.7 vs. $7.8 \%$ ).

\section{Discussion}

In our analysis of data from a registry in a primary $\mathrm{PCI}$ hospital in Jakarta, we found that the hospital admission for patients with STEMI has dramatically declined during the COVID-19 outbreak. Importantly, the DTD was longer, but the achievement of final TIMI 3 flow and in-hospital mortality were similar compared with before the COVID-19 outbreak, and deserve further discussion.

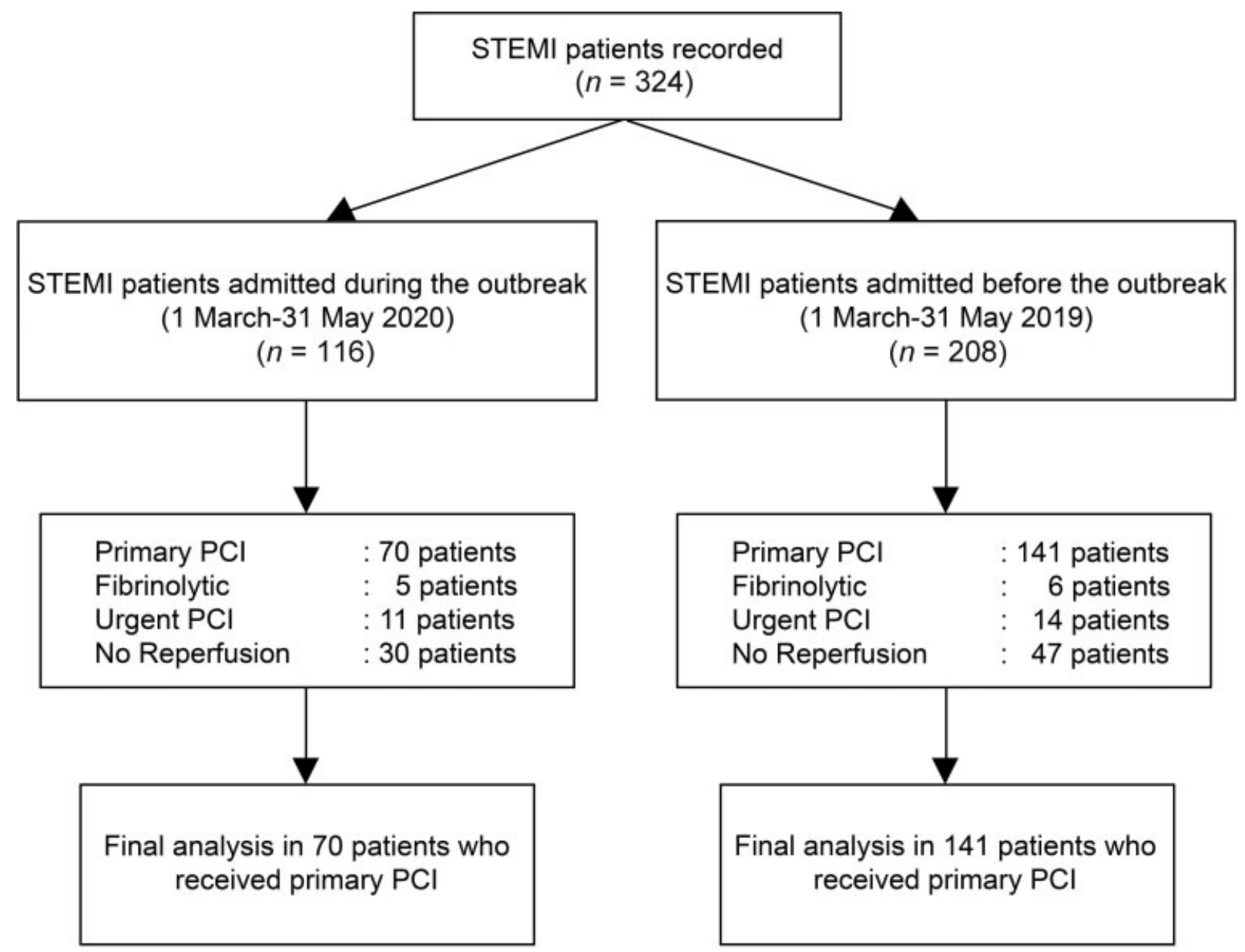

Fig. 1 Patients included in the analysis. STEMI, ST-segment elevation myocardial infarction; PCI, percutaneous coronary intervention. 
Table 1 Characteristics of patients with acute STEMI treated by primary PCI $(n=211)$

\begin{tabular}{|c|c|c|c|c|}
\hline Variables & $\begin{array}{l}\text { Missing } \\
\text { data, } n(\%)\end{array}$ & $\begin{array}{l}\text { During the } \\
\text { outbreak }(n=70)\end{array}$ & $\begin{array}{l}\text { Before the } \\
\text { outbreak }(n=141)\end{array}$ & p-Value \\
\hline Age, y & 0 & $55(46-60)$ & $54(48-59)$ & 0.43 \\
\hline Male, $n(\%)$ & 0 & $64(91.4)$ & 131 (92.9) & 0.70 \\
\hline Off-hour admission, $n(\%)$ & 0 & $31(44.3)$ & $70(49.6)$ & 0.46 \\
\hline Anterior MI, $n(\%)$ & 0 & $37(52.9)$ & $82(58.2)$ & 0.46 \\
\hline \multicolumn{5}{|l|}{ Symptom onset, $n(\%)$} \\
\hline$\leq 2 \mathrm{~h}$ & 0 & $2(2.9)$ & $9(6.4)$ & 0.34 \\
\hline$>2-6 h$ & 0 & $36(51.4)$ & $71(50.4)$ & 0.88 \\
\hline $6-12 \mathrm{~h}$ & 0 & $28(40)$ & $56(39.7)$ & 0.96 \\
\hline$>12 \mathrm{~h}$ & 0 & $4(5.7)$ & $5(3.5)$ & 0.48 \\
\hline \multicolumn{5}{|l|}{ Cardiovascular risk factors, $n(\%)$} \\
\hline Smoker & 0 & $25(35.7)$ & $92(65.2)$ & $<0.001$ \\
\hline Hypertension & 0 & $34(48.6)$ & $84(59.6)$ & 0.13 \\
\hline Diabetes mellitus & 0 & $19(27.1)$ & 55 (39) & 0.08 \\
\hline Dyslipidemia & 0 & $11(15.7)$ & $23(16.3)$ & 0.91 \\
\hline Family history & 0 & $8(11.4)$ & $12(8.5)$ & 0.49 \\
\hline \multicolumn{5}{|l|}{ Source of referral, $n$ (\%) } \\
\hline Interhospital referral & 0 & $24(34.3)$ & $94(66.7)$ & $<0.001$ \\
\hline \multicolumn{5}{|l|}{ Killip classification, n (\%) } \\
\hline I & 0 & $62(88.6)$ & $116(82.3)$ & 0.23 \\
\hline II & 0 & $4(5.7)$ & $15(10.6)$ & 0.23 \\
\hline III & 0 & $2(2.9)$ & $4(2.8)$ & 1.0 \\
\hline IV & 0 & $2(2.9)$ & $6(4.3)$ & 1.0 \\
\hline TIMI risk score $>4, n(\%)$ & 0 & $21(30)$ & $44(31.2)$ & 0.85 \\
\hline \multicolumn{5}{|l|}{ Antiplatelet therapy within 24 h, $n$ (\%) } \\
\hline Salicylic acid & 0 & $69(98.6)$ & $140(99.3)$ & 1.0 \\
\hline Clopidogrel & 0 & $56(80)$ & $110(78)$ & 0.74 \\
\hline \multicolumn{5}{|l|}{ Blood tests } \\
\hline Hemoglobin $(\mathrm{g} / \mathrm{dL})$ & 0 & $14.7(12.975-15.625)$ & $14.6(13.3-15.45)$ & 0.84 \\
\hline Leukocyte $(/ \mu \mathrm{L})$ & 0 & $13,102.5(10,880-15,402)$ & $13,300(10,780-16,080)$ & 0.69 \\
\hline Initial troponin T (ng/L) & 15 (7.109) & $323.5(106-1241)$ & $273(66.5-840)$ & 0.84 \\
\hline Creatinine level (mg/dL) & 0 & $0.94(0.825-1.1)$ & $1.03(0.85-1.245)$ & 0.006 \\
\hline Admission blood glucose, (mg/dL) & $1(0.4734)$ & $133(120.75-55.75)$ & $147(122.5-214.5)$ & 0.005 \\
\hline \multicolumn{5}{|l|}{ Echocardiography variables } \\
\hline LVEF (\%) & $21(9.952)$ & $45(35-54)$ & $45(36-51)$ & 0.76 \\
\hline \multicolumn{5}{|l|}{ Primary $\mathrm{PCl}$ procedural characteristics } \\
\hline Balloon predilatation, $n$ (\%) & 0 & $69(98.6)$ & $130(92.2)$ & 0.06 \\
\hline Use of drug-eluting stent, $n(\%)$ & 0 & $67(95.7)$ & $134(95)$ & 1.0 \\
\hline Transradial access, $n(\%)$ & 0 & $63(90)$ & $126(89.4)$ & 0.88 \\
\hline \multicolumn{5}{|l|}{ Infarct-related artery, $n$ (\%) } \\
\hline Left main & 0 & $1(1.4)$ & 0 & 0.33 \\
\hline Left anterior descending & 0 & $38(54.3)$ & $83(58.9)$ & 0.52 \\
\hline Left circumflex & 0 & $2(2.9)$ & $4(2.8)$ & 1.0 \\
\hline Right coronary artery & 0 & $29(41.4)$ & $54(38.3)$ & 0.66 \\
\hline
\end{tabular}


Table 1 (Continued)

\begin{tabular}{|l|l|l|l|l|}
\hline Variables & $\begin{array}{l}\text { Missing } \\
\text { data, } \boldsymbol{n}(\%)\end{array}$ & $\begin{array}{l}\text { During the } \\
\text { outbreak }(\boldsymbol{n}=\mathbf{7 0})\end{array}$ & $\begin{array}{l}\text { Before the } \\
\text { outbreak }(\boldsymbol{n}=141)\end{array}$ & $p$-Value \\
\hline Coronary artery involvement, $\boldsymbol{n}(\%)$ & & & & \\
\hline Single vessel disease & 0 & $32(45.7)$ & $55(39)$ & 0.35 \\
\hline Two vessel disease & 0 & $21(30)$ & $35(24.8)$ & 0.42 \\
\hline Three vessel disease & 0 & $17(24.3)$ & $51(36.2)$ & 0.08 \\
\hline Left main disease & 0 & $2(2.9)$ & $8(5.7)$ & 0.5 \\
\hline Medication at discharged, $\boldsymbol{n}(\%)$ & & & & \\
\hline Salicylic acid & 0 & $65(92.9)$ & $130(92.2)$ & 0.86 \\
\hline Clopidogrel & 0 & $46(65.7)$ & $103(73)$ & 0.27 \\
\hline ACE/ARB inhibitors & 0 & $58(82.9)$ & $122(86.5)$ & 0.47 \\
\hline Beta blocker & 0 & $59(84.3)$ & $119(84.4)$ & 0.98 \\
\hline Simvastatin & 0 & $65(92.9)$ & $130(92.2)$ & 0.86 \\
\hline Length of stay, d & 0 & $5(4-7)$ & $5(4-6)$ & 0.55 \\
\hline
\end{tabular}

Abbreviations: ACE, angiotensin converting enzyme; ARB, angiotensin receptor blocker; LVEF, left ventricular ejection fraction; MI denotes myocardial infarction; NA, not analyzed; $\mathrm{PCl}$, percutaneous coronary intervention; TIMI, thrombolysis in myocardial infarction.

The results of this study suggest that it is still feasible to perform primary PCI during the COVID-19 pandemic with expected delays in reperfusion time that partly due to the COVID-19 screening at the ED. However, the delays should not reduce the utilization of primary $\mathrm{PCI}$, indicating that during the COVID-19 pandemic, primary PCI should continue to be the standard of care for patients with definite STEMI, particularly when patients are presenting to a primary $\mathrm{PCI}$ center. However, during the COVID-19 pandemic, all primary PCI procedures should be performed in the way of safest environment, indicating that all patients with STEMI should be treated as possible COVID-19 at presentation.

A recent protocol from China ${ }^{11}$ suggests use of fibrinolytic therapy as the initial treatment for patients with acute STEMI within 12 hours of symptom onset during the pandemic. However, the protocol should not be generalized to all STEMI care during the pandemic since the success rate of fibrinolytic therapy in restoring the flow of the IRA is lower than primary $\mathrm{PCI}$, and this was associated with a worse clinical outcome in patients who receive fibrinolytic therapy as compared with primary PCI. ${ }^{12}$ However, in some cases of highly expected delays for primary PCI due to COVID-19 assessment or establishing the STEMI diagnosis, it is reasonable to initiate fibrinolytic therapy, particularly for those patients who first presented to a non-PCI center with possible prolonged door-in to door-out time. In our study, fibrinolytic therapy was successfully initiated in four patients during the study period. Fibrinolytic therapy was indicated due to the expected delay for primary $\mathrm{PCI}$ related to the COVID-19 assessment at the ED, and was administered during the early weeks of the COVID-19 outbreak.

In our study, the DTD time was longer during the COVID19 outbreak that related to the COVID-19 screening at the ED including the epidemiology screening, chest X-ray, severe acute respiratory syndrome coronavirus 2 antibody evaluation, and additional blood test measurement (lymphocyte and C-reactive protein). The COVID-19 screening at the ED should be effective and efficient, and this can be achieved by

Table 2 Study outcomes

\begin{tabular}{|l|l|l|l|}
\hline & $\begin{array}{l}\text { During the outbreak } \\
(\boldsymbol{n}=\mathbf{7 0})\end{array}$ & $\begin{array}{l}\text { Before the outbreak } \\
(\boldsymbol{n}=\mathbf{1 4 1})\end{array}$ & $p$-Value \\
\hline Symptom onset-to-PCI hospital admission, min & $360(240-540)$ & $360(270-480)$ & 0.84 \\
\hline Door-to-device time, min & $104(79.50-149.25)$ & $81(67-111)$ & $<0.001$ \\
\hline Total ischemic time, min & $475.5(354-679.5)$ & $449(356.5-585.5)$ & 0.43 \\
\hline Final TIMI flow, $n$ (\%) & & & \\
\hline 0 & $1(1.4)$ & $1(0.7)$ & 1.0 \\
\hline 1 & $0(0)$ & $1(0.7)$ & 1.0 \\
\hline 2 & $8(11.4)$ & $16(11.3)$ & 0.98 \\
\hline 3 & $61(87.1)$ & $123(87.2)$ & 0.98 \\
\hline In-hospital mortality, $n(\%)$ & $4(5.7)$ & $11(7.8)$ & 0.77 \\
\hline
\end{tabular}


a good collaboration between the staff at the ED, imaging, laboratory department, and the interventional cardiologists on duty. In certain circumstances, there will be longer time needed to assess the COVID-19 possible such as in patients with unclear STEMI diagnosis that potentially increases the delay in transferring the patient to the catheterization laboratory that also leads to a longer DTD time. Early activation of the catheterization laboratory is also crucial in maintaining the recommended DTD time during the pandemic to prepare the safest environment during the interventional procedures. In our center, all staff involved in the interventional procedure followed the use of standard personal protective equipment including use of gown, N95, face shield, and covered shoes. We also used a dedicated catheterization laboratory room to perform primary PCI cases during the study period. It is also suggested to avoid unnecessary X-ray projections during coronary angiography of primary $\mathrm{PCI}$ to minimize the total procedural time. In addition, the number of interventional cardiologists in the hospital should be appropriate to allow the operator working within shift and avoid excessive exposure to the primary PCI procedures. Currently, there are 15 interventional cardiologists who are responsible for primary $\mathrm{PCI}$ in the hospital that work within a daily shift and to ensure adequate time for recovery, and perhaps related to the high achievement of TIMI 3 flow after PCI during the COVID-19 outbreak in 90\% of cases, and this was similar to the achievement of before the outbreak (-Table 2 ).

During the COVID-19 outbreak, the utilization of primary PCI in our hospital was lower than before the outbreak (51.4 vs. $74 \%$ ), and this was partly associated with an overall $41 \%$ drop of the STEMI admissions in the hospital, whereas admission through interhospital hospital reduced by $74 \%$ (-Table 1). Other possible reasons for the lower uptake of primary $\mathrm{PCI}$ in the hospital during the COVID-19 outbreak were related to patients who had complete resolution of STsegment elevation at presentation, thus treated conservatively. Several speculations were described to explain the dramatic reduction in admission of STEMI patients from interhospital transfer in the region during the COVID-19 outbreak such as people are afraid of getting infected by COVID-19 when visiting the EDs of the primary hospitals, and the strict regulations to stay and work from home, social distancing, large scale social restriction, and self-isolation may partly prevent patients going to hospital to seek help for their health conditions.

A recent recommendation emphasizes the use of primary $\mathrm{PCI}$ in definite STEMI patients, and primary PCI should be the standard of care for STEMI patients during the COVID-19 pandemic with some important caveats, and all patients presenting with a suspected STEMI should be considered as COVID-19 positive, ${ }^{13}$ which is a reasonable guide for our daily clinical practice during this pandemic.

All health-care providers should prepare for the unexpected arrival of the delayed presentation of patients with ACS during the pandemic, which are probably associated with severe complications such as acute heart failure, arrhythmia, and mechanical complications. Furthermore, it also important that all hospitals should prepare for the possible increase in hospital visits by patients with cardiovascular disease soon after the COVID-19 outbreak has been controlled.

Finally, the regional systems of care for STEMI patients should be able to provide a simple and efficient protocol to transfer patients from non-PCI hospitals to a primary $\mathrm{PCI}$ hospital within the reasonable time delay, and if possible introduce the fibrinolytic therapy protocol immediately after admission at the non-PCI centers in patients with STEMI who presented early after symptom onset, before transferring the patient to a PCI center.

\section{Study Limitation}

This study has several limitations. First, this was a singlecenter study with relatively small sample size. Second, we did not record the time delay at the first hospital before the transfer process in patients who admitted from interhospital transfer process. Finally, we did not evaluate other systemrelated delay at our hospital including the time from ECG recording to STEMI diagnosis, and from STEMI diagnosis to catheterization arrival.

\section{Conclusion}

During the COVID-19 outbreak, we found a longer DTD time for primary angioplasty, but the achievement of final TIMI 3 flow and in-hospital mortality were similar as compared with before the COVID-19 outbreak. Thus, primary angioplasty should remain the standard of care for STEMI during the COVID-19 outbreak, and it should be performed with several precautions and within the safest environment.

\section{Note}

There was no external funding to support this study, and this study has no relationship with any industrial company.

\section{Disclosures \\ Nothing to declare.}

\section{Funding}

No external funding support.

\section{Conflict of Interest}

None.

\section{Acknowledgments}

We thank all the personnel including the physicians and nurses who dedicated their work during the COVID-19 outbreak at the emergency department, catheterization laboratory, and intensive cardiovascular care unit of the National Cardiovascular Center Harapan Kita, Jakarta.

\section{References}

1 De Filippo O, D'Ascenzo F, Angelini F, et al. Reduced rate of hospital admissions for ACS during Covid-19 outbreak in Northern Italy. N Engl J Med 2020;383(01):88-89 
2 Metzler B, Siostrzonek P, Binder RK, Bauer A, Reinstadler SJ. Decline of acute coronary syndrome admissions in Austria since the outbreak of COVID-19: the pandemic response causes cardiac collateral damage. Eur Heart J 2020;41(19):1852-1853

3 Dharma S, Andriantoro H, Purnawan I, et al. Characteristics, treatment and in-hospital outcomes of patients with STEMI in a metropolitan area of a developing country: an initial report of the extended Jakarta Acute Coronary Syndrome registry. BMJ Open 2016;6(08):e012193. Doi: 10.1136/bmjopen-2016-012193

4 Dharma S, Andriantoro H, Dakota I, et al. Organisation of reperfusion therapy for STEMI in a developing country. Open Heart 2015; 2(01):e000240. Doi: 10.1136/openhrt-2015-000240

5 Dharma S, Siswanto BB, Firdaus I, et al. Temporal trends of system of care for STEMI: insights from the Jakarta Cardiovascular Care Unit Network System. PLoS One 2014;9(02):e86665. Doi: 10.1371/journal. pone.0086665

6 Dharma S, Sari NY, Santoso A, Sukmawan R, Rao SV. Association of plasma pentraxin 3 concentration with angiographic and clinical outcomes in patients with acute ST-segment elevation myocardial infarction treated by primary angioplasty. Catheter Cardiovasc Interv 2020;96(06):1233-1239

7 Central Bureau of Statistics - Indonesia. 2016 statistics

8 Jakarta Tanggap COVID-19. Dinas Kesehatan DKI Jakarta ; [cited 2020 April 27]. Accessed March 11, 2021 from: https://corona. jakarta.go.id/id
9 Ibanez B, James S, Agewall S, et al; ESC Scientific Document Group. 2017 ESC Guidelines for the management of acute myocardial infarction in patients presenting with ST-segment elevation: The Task Force for the management of acute myocardial infarction in patients presenting with ST-segment elevation of the European Society of Cardiology (ESC). Eur Heart J 2018;39(02):119-177

10 O'Gara PT, Kushner FG, Ascheim DD, et al; CF/AHA Task Force. 2013 ACCF/AHA guideline for the management of ST-elevation myocardial infarction: executive summary: a report of the American College of Cardiology Foundation/American Heart Association Task Force on Practice Guidelines. Circulation 2013;127 (04):529-555

11 Zeng J, Huang J, Pan L. How to balance acute myocardial infarction and COVID-19: the protocols from Sichuan Provincial People's Hospital. Intensive Care Med 2020;46(06):1111-1113

12 Keeley EC, Boura JA, Grines CL. Primary angioplasty versus intravenous thrombolytic therapy for acute myocardial infarction: a quantitative review of 23 randomised trials. Lancet 2003; 361(9351):13-20

13 Mahmud E, Dauerman HL, Welt FGP, et al. Management of acute myocardial infarction during the COVID-19 pandemic: a position statement from the Society for Cardiovascular Angiography and Interventions (SCAI), the American College of Cardiology (ACC), and the American College of Emergency Physicians (ACEP). J Am Coll Cardiol 2020;76(11):1375-1384 